\section{The highest energy}

\section{particles in nature}

\author{
A. A. Watson \\ Department of Physics and Astronomy, University of Leeds \\ Leeds LS2 9JT, UK
}

$T$ he highest energy particles in Nature are cosmic rays. The most energetic one yet detected has an energy of $3 \times 10^{20} \mathrm{eV}$, a macroscopic energy greater than that of a tennis ball travelling at $250 \mathrm{~km} \mathrm{~h}^{-1}$. This energy is probably not set by Nature but by the relatively small area $\left(\sim 100 \mathrm{~km}^{2}\right)$ of the devices that have been used to search for them thus far as the rate above $10^{20} \mathrm{eV}$ is only about 1 per $\mathrm{km}^{2}$ per century. We do not know how these particles are created, or whether they are photons, atomic nuclei or neutrinos, but experiments underway promise data that may lead us to new understandings of some important astrophysical problems - or even to some new physics.

That we know anything about such extraordinary particles is because of searches that were started for the origin of much lower energy cosmic rays many years ago. In 1938 the French scientist, Pierre Auger, discovered serendipitously that showers of particles, secondaries created in the atmosphere by an incoming cosmic ray, were spread out over distances of $300 \mathrm{~m}$ at ground level. The energy of the initiating particles was estimated to be about $10^{15} \mathrm{eV}$. The particles making up the shower travel through the atmosphere at the velocity of light and are confined to a relatively thin disc, rather like a giant dinner plate. In the 1950s it became possible to find the arrival direction of such particles by measuring the relative arrival times of the shower disc at detectors placed on a widely spaced grid. The detectors of choice have usually been plastic scintillators or water-Cherenkov detectors and with these the direction of the incoming primaries can be measured to about $1^{\circ}$. A shower produced by a cosmic ray of $10^{20}$ $\mathrm{eV}$ contains about $10^{11}$ particles at ground level spread out over an area of about $20 \mathrm{~km}^{2}$.

\section{Are there particles with an energy above $5 \times 10^{19} \mathrm{eV}$ ?}

The Larmor radius of a proton of $10^{18} \mathrm{eV}$ in the galactic magnetic field is about $0.3 \mathrm{kpc}$. This is comparable to the thickness of the galactic disc and hence it was expected that some features of galactic structure could be mapped out in 'cosmic ray light' if a sufficient number of high-energy particles could be collected. Over the years shower arrays of greater and greater area have been constructed to detect the rare, energetic events but no convincing evidence of an anisotropy or point source of cosmic rays has been found. Although the 'astronomy' of cosmic rays may thus seem a little dull, this turns out to be part of the puzzle about their origin. Indeed the search for sources might well have ended by now had it not been for the discovery of the $2.7 \mathrm{~K}$ cosmic background radiation. In 1966 Greisen and Zatsepin and Kuzmin pointed out that there might be a cut-off (the GZK cutoff) in the energy spectrum at about $5 \times 10^{19} \mathrm{eV}$. By 1966 many events above $10^{18} \mathrm{eV}$ had been detected (with one claimed to be of $10^{20} \mathrm{eV}$ ). The view was commonly held that the failure to discover point sources, combined with rather general arguments about source energetics, could most readily be explained if the particles were produced in unusual objects far from our galaxy: quasars, also little understood at the time, were thought to satisfy the requirements. If this was so then sufficiently energetic protons (which see the $2.7 \mathrm{~K}$ photons Doppler shifted by a factor proportional to their Lorentz factor) lose energy through photopion production and their energies decrease significantly as they travel through space. A similar argument, but invoking photoproduction, applies to heavy nuclei. Calculations showed that a $10^{20} \mathrm{eV}$ proton has only a $50 \%$ chance of originating further than $20 \mathrm{Mpc}$ from Earth. A few very powerful objects (e.g. Cen A and M87) lie within this distance but the most energetic cosmic rays do not point back to them.

\section{Large ground-based detectors will search for ultra high- energy cosmic rays}

The existence, or not, of the GZK cut-off has not been easy to establish. The early experiments at Volcano Ranch (US), Haverah Park (UK) and Yakutsk (Russia) had insufficient area to detect more than a very few particles above the cut-off energy (which is very precisely known because of the blackbody nature of the 2.7 $\mathrm{K}$ spectrum and the accurate cross-sections of the interaction processes). Furthermore the need to estimate the energies using Monte Carlo calculations, in which many of the simulated interactions are unstudied by man-made accelerators, made many suspicious of the claims. Fortunately another detection method was invented and bccame technologically feasible in the late 1970s. This technique uses the fact that the Earth's atmosphere responds to the passage of a shower by emitting fluorescence radiation in the UV part of the spectrum. If the shower is large enough the radiation can be detected with a photomultiplier camera on clear moonless nights and the growth and decay of the cascade can be followed and measured. A model-independent

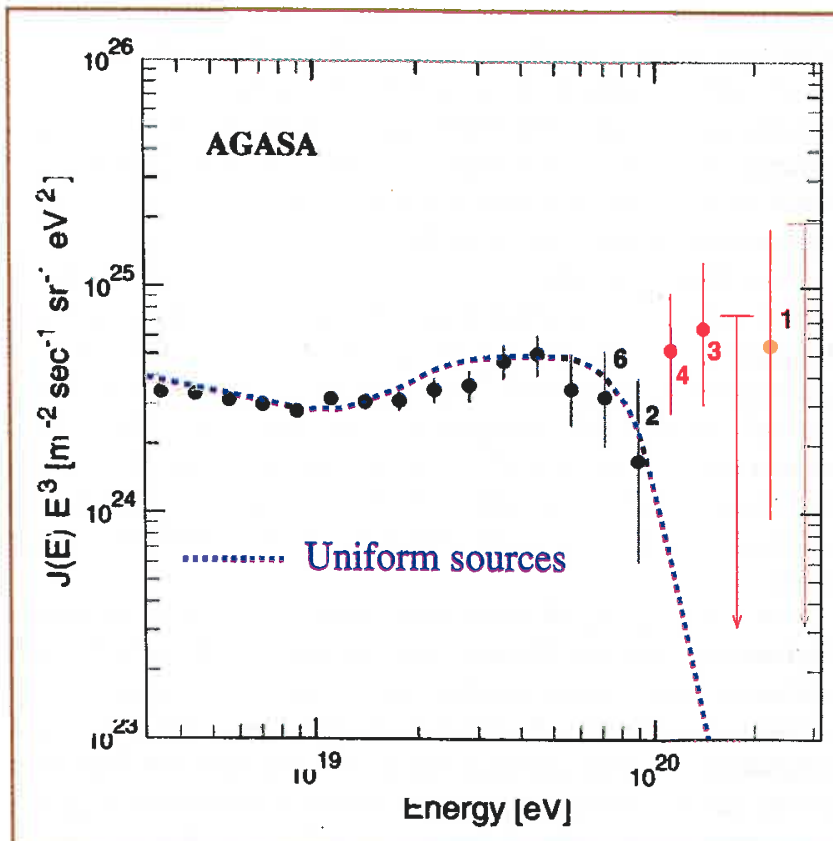

Fig. 1: The energy spectrum as measured by the Japanese shower array known as AGASA. This array comprises $111 \times 2.2 \mathrm{~m}^{2}$ scintillators spread over $100 \mathrm{~km}^{2}$. The data shown are from Takeda et al. (1998). The dashed line indicates the spectrum that would be expected if the ultra high-energy cosmic rays were produced in sources distributed uniformly throughout the Universe, with due allowance for measurement uncertainty. The predicted GZK cut-off, due to photo-pion production on the $2.7 \mathrm{~K}$ radiation field is evident in the theoretical curve but clearly absent in the data. 
estimate of the energy is in principle feasible so that energies can be measured in a manner similar to the calorimetery approach widely used at particle accelerators. Although many fewer events have been detected by this technique than by the surface arrays a key conclusion is that the fluxes in the critical energy region are very similar. This fact, and the discovery of an event of $3 \times 10^{20} \mathrm{eV}$ in 1993 by the fluorescence group at the University of Utah, have led most people to believe that there are particles at energies well above the GZK cut-off. A striking result, from the $100-\mathrm{km}^{2}$ shower array operated by the Japanese AGASA group, is shown in figure 1. With this instrument 8 events have been detected above $10^{20} \mathrm{eV}$. The data lie well above the prediction made of the spectrum if all of the particles are produced in sources distributed uniformly throughout the Universe.

The existence of events above the GZK cut-off means that the particles must be produced nearby but then why are no point sources seen? This is the enigma of ultra high-energy cosmic rays and hundreds of papers have been written suggesting explanations. The proposals range through modifications to the properties of known particles, speculation about new particles (including massive super-heavy relics created $10^{-35} \mathrm{~s}$ after the big bang), radical revisions of our understanding of the magnetic fields in space and even the breakdown of Lorentz invariance. Some argue that the highest energy particles might be iron nuclei. Certainly this would explain the isotropy, as the iron nuclei are more readily bent by the magnetic field, but it is not clear that they would survive the photon field surrounding likely sources.

In an effort to solve the enigma observatories with greater apertures are being developed. A second-generation fluorescence detector, known as HiRes, has been constructed by the Utah group and data from a significant exposure as now been reported Currently the group have failed to confirm the rate of events above $10^{20} \mathrm{eV}$ seen by the AGASA group but there are unanswered questions about the efficiency of the fluorescence mechanism for turning electron energy into ultra violet photons. The HiRes group do see some events above $10^{20} \mathrm{eV}$, well beyond the GZK cut-off. A more complex device, that blends the strengths of ground arrays and fluorescence detectors, is under construction at Malargue in Mendoza Province, Argentina. Scientists from 18 countries, including several in Europe, are working on this project. We are building a hybrid instrument of 1600 water-Cherenkov tanks spread over $3000 \mathrm{~km}^{2}$ (about 30 times the area of the city of Paris) with four fluorescence detectors over-looking the site. There will be dual measurements of the showers in $10 \%$ of events so that calibration of the energy deduced from the surface detectors will be possible. Construction of this instrument, the Pierre Auger Observatory, is underway and the prototype fluorescence unit and the surface array have already detected showers. Two of the water-tanks are shown in figure 2 . The full instrument should be completed by late 2004 .

\section{Plans for observing atmospheric fluorescence from space}

To achieve an exposure greater than that targeted by the Auger Observatory may require putting a detector into space. The idea, proposed by Linsley, who was also the first to claim detection of a $10^{20} \mathrm{eV}$ event, is to observe the fluorescence light using a detector mounted in a satellite. As a first stage an instrument with $2 \times 10^{5}$ photomultipliers has been designed for the International Space Station and is the subject of an ESA phase-A study. Called EUSO, for Extreme Universe Space Observatory, it will be come into operation in 2007 and should detect 3000 events above $10^{20} \mathrm{eV}$ in

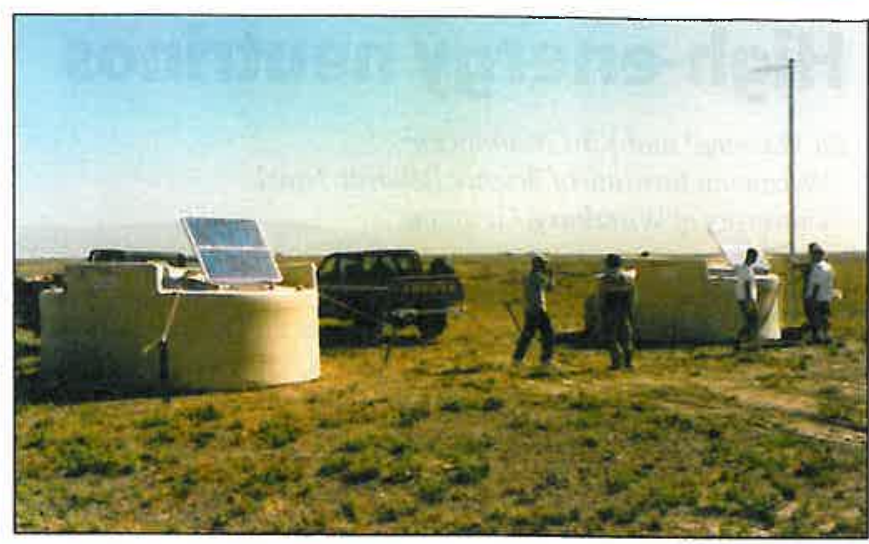

Fig. 2: Two of the tanks in the Pierre Auger Observatory are shown. They each hold 12 tonnes of clean water and are viewed by $3 \times 8^{\prime \prime}$ diameter photomultipliers. The electronics for recording and data transmission are powered by solar cells. These tanks are placed close together so that cross-tank measurements of densities and arrival times can be made but the nearest neighbour for all other tanks is $1.5 \mathrm{~km}$ away. In this way $3000 \mathrm{~km}^{2}$ can be covered with only 1600 detectors.

its three-year lifetime. This exceeds the 500 events anticipated from the Auger Observatory from 10 years of operation and the 50 or so events expected from HiRes in a similar period. Again an understanding of the fluorescence emission process is of major importance.

Of the many ideas that will be tested with data from the Auger Observatory, and from EUSO, one is the proposal that Cen A, a very powerful radio galaxy only 3.4 Mpc from the Earth, might be an ultra high-energy cosmic ray source. It is argued by some that we have not seen it because the magnetic field in the space between Cen $A$ and us is stronger than most astronomers believe. However it has also been shown that photo-pion production within Cen A could create neutrons. These live long enough, and could be sufficiently numerous, to give a clear signal. Perhaps a point source of cosmic rays will at last be seen and the problem of the origin of the highest energy particles in Nature will have been, at least partially, solved.

\section{Bibliography}

M. Takeda et al., Phys. Rev. Lett. 1998, 81, 1163 (figure 1)

M. Nagano and A.A. Watson, Rev. Mod. Phys. 2000, 72, 689 (broad review)

The HiRes detector: www.cosmic-ray.org/

The Pierre Auger Observatory: www.auger.org/

The EUSO project: www.ifcai.pa.cnr.it/ EUSO/

\section{About the author}

Alan Watson did his $\mathrm{PhD}$ at the University of Edinburgh where he studied the processes of condensation of water vapour. He has worked in the field of high-energy cosmic rays since moving to the University of Leeds in 1964. He was heavily involved in the construction and operation of the $12-\mathrm{km}^{2}$ Haverah Park array, built near Leeds, until its closure in 1987. Subsequently he lead a team from the UK and the USA that built a shower array at the South Pole to look for high-energy gamma rays from the supernova SN1987A. Currently he is co-spokesperson for the Pierre Auger Observatory and was, with Jim Cronin (Chicago) one of the early drivers for this project. He was elected to the Royal Society in 2000. 